\title{
Dermatological Manifestations in Patients with COVID-19 Pneumonia in Veracruz, Mexico
}

\author{
Luis Del Carpio-Orantes ${ }^{1 *}$ (D), Sergio García-Mendez², Jesús Salvador Sánchez-Díaz ${ }^{3}$, Karla Gabriela \\ Peniche-Moguel', Elisa Estefanía Aparicio-Sánchez ${ }^{1}$, Orlando Israel Segura-Rodríguez ${ }^{1}$, Andrés Aguilar- \\ Silva ${ }^{1}$, Omar García-Hernández ${ }^{1}$, Alejandro Escobar-Huerta ${ }^{1}$, Azael Ahumada-Zamudio ${ }^{1}$, Andrés \\ Realino Velasco-Caicero ${ }^{1}$, Olga González-Segovia ${ }^{1}$ and Ernesto Javier Pacheco-Pérez ${ }^{1}$
}

\author{
${ }^{1}$ Department of Internal Medicine, General Hospital 71, North Veracruz Delegation, Instituto Mexicano del \\ Seguro Social, Veracruz, Mexico \\ ${ }^{2}$ Head of Teaching and Research, Regional Hospital of High Specialty of Oaxaca, Oaxaca, Mexico \\ ${ }^{3}$ Critical Care Unit, High Specialty Medical Unit 189, North Veracruz Delegation, Instituto Mexicano del \\ Seguro Social, Veracruz, Mexico
}

\section{Introduction}

A large number of viral infections are characterized by the presence of cutaneous manifestations. Multiple dermatological manifestations have been observed in patients with COVID-19, however, among the main challenges that dermatology faces is the difficult relationship between the appearance of dermatological lesions with the infection itself, as well as the poor description of the lesions that are normally determined only as "rash" [1-4].

Dermatological lesions around COVID-19 infection can be classified into three main groups:

- Dermatological lesions related to protective equipment and hygiene measures: The skin manifestations in health workers are mainly caused by friction, hyperhydration effects and contact reactions [5].

- Dermatological lesions observed in SARS-CoV-2 virus infection.

- Dermatological lesions derived from the treatment of COVID-19 [2].

Among the main dermatological lesions observed in patients with SARS-Cov-2 infection are the following:

$>$ Morbilliform/confluent erythematous/maculopapular rash: Rashes are the most commonly encountered lesions. It predominantly affects the trunk, it is considered the most common skin manifestation. It is usually observed at the beginning or after hospital discharge. Maculopapular lesions are very rare $[1,6]$.

Acral lesions similar to the pernio: Also known as "Covid fingers" that are observed as erythematous-violaceous or purpuric macules on fingers, elbows, toes with or without accompanying edema and itching in the absence of exposure to cold or renal situations to the appearance of pernio. They resolve in 4 to 8 weeks. A virus-induced vascular injury is suggested as the cause of these injuries. It has been observed in studies of the American Academy of Dermatology and Spanish the appearance of pernia lesions after symptoms by COVID-19 in which the PCR is negative and IgG and IgM positivity, which orients to a postviral or late-onset process, although it is not specific since it can also be observed in patients with positive PCR. Corticosteroids may be used if the lesions cause discomfort [1].

- Fixed livedo reticularis/livedo racemosa-like lesions: Livedo reticularis was observed in $6 \%$ of the patients. It has been speculated that these skin manifestations may be the result of small vessel occlusions whose pathogenesis mechanism remains unknown. Both livedo reticularis/ racemosa-like lesions such as purpura and necrotic lesions have been seen predominantly in elderly patients with severe systemic symptoms and a higher mortality rate [6].

> Retiform purpura/necrotic vascular lesions: Thrombotic vasculopathy and coagulation disorders have also been observed in patients with severe COVID-19. Purpura and necrotic vascular lesions were separated from livedo re-

*Corresponding author: Luis Del Carpio Orantes, Department of Internal Medicine, General Hospital 71, North Veracruz Delegation, Instituto Mexicano del Seguro Social, Veracruz, Mexico, Tel: 2292237032

Accepted: December 26, 2020

Published online: December 28, 2020

Citation: Carpio-Orantes LD, García-Mendez S, Sánchez-Díaz JS, et al. (2020) Dermatological Manifestations in Patients with COVID-19 Pneumonia in Veracruz, Mexico. Dermatol Arch 4(1):112-114 
ticularis and racemosa, taking into consideration that the aforementioned would have an origin of vasculopathy while purpura and necrotic lesions develop as vasculitis [1,6].

Urticaria: The relationship of urticaria with viral infections is well known, so COVID-19 infection should be considered a possible etiology of urticaria. The AAD (American Academy of Dermatology) has initiated an international registry to identify dermatological lesions associated with COVID-19, within which urticaria is considered in third place. The AAD (American Academy of Dermatology) Two large consensus groups (EAACI/WAO and AAAAI/ACCA) agreed on the use of short-term corticosteroids as an appropriate treatment for urticarial exacerbations [7].

Vesicular rash (similar to chickenpox): In a series of 24 patients, it was determined that the vesicular eruption associated with COVID-19 has 2 different presentation patterns: Diffuse and localized. The diffuse pattern is polymorphic and has a tendency towards widespread distribution, while the localized one is monomorphic and involves only the trunk. It usually appears after the onset of typical COVID-19 symptoms, although it can also appear after them. Dermatological lesions are not related to antivirals or other treatments. PCR does not detect the presence of SARS-CoV-2 within the vesicles $[8,9]$.

Multisystemic inflammatory syndrome in children: Polymorphic erythematous eruption, erythema or firm induration of the hands and feet, oral mucositis and conjunctivitis along with systemic laboratory and imaging findings of atypical severe Kawasaki disease have been reported in an Italian cohort study and have been observed in the United Kingdom, the United States and other countries [1]. The reported cases of patients with Kawasaki disease presented with laboratory studies and characteristic symptoms of COVID-19, some of which had a positive PCR for SARS-CoV-2; however, the relationship between both diseases has not yet been fully defined [9].

Dermatological lesions in patients infected by SARS-CoV-2 such as livedo reticularis, rash and vascular lesions may represent manifestations of secondary phenomena such as paraviral rashes or by participation of the innate or adaptive immune system that cause vasodilation, vascular leakage or procoagulant effects [10].

A recent Italian study shows that $20.4 \%$ of skin manifestations in patients with COVID-19 are in the form of erythematous rash, urticaria, and vesicular rash without these having a direct relationship with the severity of the respiratory problem [3].

No dermatological lesions have been described in Mexico associated with COVID-19, so we made a description of lesions in a group of patients from Veracruz, Mexico.

\section{Material and Methods}

Descriptive and observational study, adult patients with COVID-19 pneumonia were selected, confirmed by RT-PCR and chest CT, who were hospitalized at the General Hospital of Zone 71 from April to June 2020 in the City of Veracruz,
Mexico. General symptoms, hematic cytometry results, pneumonia severity, prognosis as well as dermatological manifestations are characterized. Confirmation was made by determining qPCR-RT for SARS-CoV-2 with the superscript III platinum One Step Quantitative RT-PCR System kit, carried out at the Central Epidemiology Laboratory of the National Epidemiological Surveillance Coordination. Non-adult patients who do not have a diagnosis confirmed by CT and RT-PCR are excluded.

\section{Results}

100 patients were entered into the study, with an average age of 49.4 years, $54 \%$ male. The general symptoms with the highest incidence were: fever, cough and dyspnea characteristic of SARS-CoV-2 infection, followed by chest pain, headache, anosmia and dysgeusia. The main alteration of the hemogram was lymphopenia, no leukopenia or plaquetopenia was demonstrated. $54 \%$ of those affected had mild pneumonia, the rest severe pneumonia. $75 \%$ progressed towards improvement and $25 \%$ died. Among the dermatological manifestations identified, all occurred in cases with severe pneumonia, the one with the highest incidence was the morbilliform viral exanthema in $18 \%$, the presence of diffuse partial alopecia in $7 \%$ as well as manifestations of lividity and maceration in $1 \%$. Regarding alopecia, in $6 \%$ it was reversible androgenetic alopecia, having manifested during the acute stage of pneumonia (all men), in $1 \%$ it presented alopecia areata (male) that has been persistent beyond the acute phase and in frank recovery (Table 1 and Table 2).

Table 1: Result of the measurement of demographic and clinical variables.

\begin{tabular}{|l|l|}
\hline Variable & $\begin{array}{l}\text { Result }^{*} \\
\mathbf{n}=100\end{array}$ \\
\hline Demographic variables & $49.4(19.3)$ \\
\hline Age, years & \\
\hline Gender; $\mathbf{n}(\%)$ & $46(46)$ \\
\hline Women & $54(54)$ \\
\hline Men & \\
\hline Laboratory variables & $10,103.0(4,289.0)$ \\
\hline Leukocytes, cel/mm ${ }^{3}$ & $8,509.3(4,216.0)$ \\
\hline Neutrophils, cel/mm ${ }^{3}$ & $1112.7(585.4)$ \\
\hline Lymphocytes, cel/mm ${ }^{3}$ & $258,548.0(127,947.2)$ \\
\hline Platelets, cel/mm ${ }^{3}$ & \\
\hline Type of CovID-19 pneumonia, & \\
\hline (\%) & $54(54)$ \\
\hline Mild pneumonia & $46(46)$ \\
\hline Severe pneumonia & $75(75)$ \\
\hline Hospital outcome, $\mathbf{n}$ (\%) & $25(25)$ \\
\hline Improvement & \\
\hline Death & \\
\hline
\end{tabular}

"Results expressed in means with standard deviation ( $\pm D E)$, except where something different is indicated

COVID-19: Coronavirus Infectious Disease - 19 
Table 2: Clinical manifestations.

\begin{tabular}{|l|l|}
\hline General Symptoms & $\mathbf{N = 1 0 0 ~ ( \% ) ~}$ \\
\hline Fever & $96 \%$ \\
\hline Cough & $95 \%$ \\
\hline Dyspnea & $85 \%$ \\
\hline Chest pain & $83 \%$ \\
\hline Headache & $83 \%$ \\
\hline Arthralgias & $80 \%$ \\
\hline Anosmia & $75 \%$ \\
\hline Dysgeusia & $75 \%$ \\
\hline Myalgias & $70 \%$ \\
\hline Drowsiness & $50 \%$ \\
\hline Diarrhea & $50 \%$ \\
\hline Abdominal pain & $40 \%$ \\
\hline Distal partesias & $10 \%$ \\
\hline Encephalopathy & $5 \%$ \\
\hline Delirium & $3 \%$ \\
\hline Dermatological manifestations & \\
\hline Morbiliform viral rash & $18 \%$ \\
\hline Alopecia & $7 \%$ \\
\hline Lívedo reticularis & $1 \%$ \\
\hline Maceration of the skin of the hands & $1 \%$ \\
\hline
\end{tabular}

\section{Discussion}

The dermatological manifestations stand out, especially alopecia, which has been considered a new manifestation of COVID-19, highlighting our data with those previously reported where, in severe forms of the disease, greater cases of androgenetic alopecia have been reported, predominantly in men; Various drugs such as steroids (methylprednisolone and dexamethasone), enoxaparin and tocilizumab were used in our patients, which probably contributed to these manifestations, although it is not entirely clear whether it was due to direct viral action or due to various interactions at the hair bulb level. With regard to steroids, these have been used as a treatment for alopecia; Alopecia associated with enoxaparin or tocilizumab is considered very rare, although there are case reports in this regard [11-14].

\section{Conclusions}

The incidence of dermatological manifestations is low in this study population, the most frequent being the morbilliform viral exanthema expected in a virus, however they present manifestations of low incidence such as reversible androgenetic alopecia associated with severity of the disease, a finding that has been documented recently as a manifesta- tion associated with COVID-19, so we consider it important to maintain close surveillance of this type of manifestation in patients affected by said pathology, since it can be a clinical manifestation that acts as a marker of severity, coupled with the use of various drugs for the treatment of the disease.

\section{References}

1. Steven R Feldman, Esther E Freeman (2020) Coronavirus disease 2019 (COVID-19): Cutaneous manifestations and issues related to dermatologic care. UpToDate.

2. Criado PR, Pagliari C, Carneiro FRO, et al. (2020) Lessons from dermatology about inflammatory responses in Covid-19. Rev Med Virol.

3. Fahmy DH, El-Amawy HS, El-Samongy MA, et al. (2020) COVID-19 and dermatology: A comprehensive guide for dermatologists. J Eur Acad Dermatol Venereol 34: 13881394.

4. Ring J (2020) COVID-19 and its implications for dermatology and venereology. J Eur Acad Dermatol Venereol 34: 914.

5. Masood S, Tabassum S, Naveed S, et al. (2020) COVID-19 pandemic \& skin care guidelines for health care professionals. Pak J Med Sci 36(COVID19-S4): S115-S117.

6. Marzano AV, Cassano N, Genovese G, et al. (2020) Cutaneous manifestations in patients with COVID-19: A preliminary review of an emerging issue. $\mathrm{Br} \mathrm{J}$ Dermatol 183: 431-442.

7. Pathania YS (2020) Urticaria and COVID-19 infection: A critical appraisal. J Dermatolog Treat.

8. Fernandez-Nieto D, Ortega-Quijano D, Jimenez-Cauhe J, et al. (2020) Clinical and histological characterization of vesicular COVID-19 rashes: A prospective study in a tertiary care hospital. Clin Exp Dermatol 45: 872-875.

9. Xu S, Chen M, Weng J (2020) COVID-19 and Kawasaki disease in children. Pharmacol Res 159: 104951.

10. Gül Ü (2020) COVID-19 and dermatology. Turk J Med Sci.

11. Criado PR, Pincelli TPH, Criado RFJ, et al. (2020) Potential interactions of SARS-CoV-2 with human cell receptors in the skin: Understanding the enigma for a lower frequency of skin lesions compared to other tissues. Exp Dermatol 29: 936-944.

12. Wambier CG, Vaño-Galván S, McCoy J, et al. (2020) Alopecia androgenética presente en la mayoría de los pacientes hospitalizados con COVID-19: El "signo de Gabrin". J Am Acad Dermatol 83: 680-682.

13. Nadesalingam K, Goodfield M, Emery P (2016) Halo naevi, vitiligo and diffuse alopecia areata associated with tocilizumab therapy. Oxf Med Case Reports 2016: omw027.

14. Wang YY, Po HL (2006) Enoxaparin-induced alopecia in patients with cerebral venous thrombosis. J Clin Pharm Ther 31: 513-517.

DOI: $10.36959 / 661 / 314$

Copyright: (C) 2020 Carpio-Orantes LD, et al. This is an open-access article distributed under the terms of the Creative Commons Attribution License, which permits unrestricted use, distribution, and reproduction in any medium, provided the original author and source are credited. 